Check for updates

Cite this: Phys. Chem. Chem. Phys., 2019, 21, 12184

Received 24th January 2019, Accepted 14th May 2019

DOI: $10.1039 / c 9 c p 00467 j$

rsc.li/pccp

\title{
Revising the common understanding of metamagnetism in the molecule-based bisdithiazolyl BDTMe compound $\dagger$
}

\author{
Clàudia Climent, (D) ab Sergi Vela, (D) ac Joaquim Jornet-Somoza (D) ad and \\ Mercè Deumal iD *a
}

\begin{abstract}
The BDTMe molecule-based material is the first example of a thiazyl radical to exhibit metamagnetic behavior. Contrary to the common idea that metamagnetism occurs in low-dimensional systems, it is found that BDTMe magnetic topology consists of a complex 3D network of almost isotropic ferromagnetic spin-ladders that are coupled ferromagnetically and further connected by some weaker antiferromagnetic interactions. Calculated magnetic susceptibility $\chi T(T)$ data is in agreement with experiment. Calculated $M(H)$ data clearly show the typical sigmoidal shape of a metamagnet at temperatures below $2 \mathrm{~K}$. The calculated critical field becomes more apparent in the $\mathrm{d} M / \mathrm{d} H(H)$ plot, being in very good agreement with experiment. Our computational study concludes that the magnetic topology of BDTMe is preserved throughout the entire experimental range of temperatures $(0-100 \mathrm{~K})$. Accordingly, the ground state is the same irrespective of the temperature at which we study the BDTMe crystal. Revising the commonly accepted understanding of a metamagnet explained as ground state changing from antiferromagnetic to ferromagnetic, the Boltzmann population of the different states is here suggested to be the key concept: at $2 \mathrm{~K}$ the ground singlet state has more weight $(24 \%)$ than at $10 \mathrm{~K}(1.5 \%)$, where excited states have an important role. Changes in the antiferromagnetic interactions that couple the ferromagnetic skeleton of BDTMe will directly affect the population of the distinct states that belong to a given magnetic topology and thus its magnetic response. Accordingly, this strategy could be valid for a wide range of bisdithiazolyl BDTcompounds whose magnetism can be tuned by means of weak antiferromagnetic interactions.
\end{abstract}

\section{Introduction}

New molecular materials with targeted physical properties are an area of current interest from both fundamental and applicative points of view. Molecule-based magnets exhibit a wide variety of bonding and structural motifs. These include one-, two-, and three dimensional (1D, 2D, and 3D, respectively) network structures by variation of metal atoms, organic radicals, and ligands. ${ }^{1}$

${ }^{a}$ Secció Quimica Física, Dept. Ciència de Materials i Quimica Física \& IQTCUB, Universitat de Barcelona, Martí i Franquès 1, E-08028 Barcelona, Spain.

E-mail:merce.deumal@ub.edu

${ }^{b}$ Departamento de Física Teórica de la Materia Condensada, Universidad Autónoma de Madrid, E-28049 Madrid, Spain

${ }^{c}$ Laboratory for Computational Molecular Design (LCMD), Institute of Chemical Sciences and Engineering, EPFL, CH-1015 Lausanne, Switzerland

${ }^{d}$ Theory Department, The Max Planck Institute for the Structure and Dynamics of Matter (MPSD), Bldg. 99 (CFEL), Luruper Chaussee 149, 22761 Hamburg, Germany

$\dagger$ Electronic supplementary information (ESI) available: Section S1. d1-d10 isolated pairs of BDTMe radicals. Section S2. Comparison between values of $J_{i}$ from our calculations and from ref. 39. Section S3. Discussion on magnetic models. See DOI: $10.1039 / \mathrm{c} 9 \mathrm{cp} 00467 \mathrm{j}$
The contemporary challenge to the synthetic chemists is to produce new compounds with pre-assigned magnetic, electrical, and optical properties and, for this, the rational synthetic design is addressed to tune the solid-state structure. The ability to manipulate magnetic coupling and/or charge transfer between spin carriers is thus the key to creating new multifunctional materials. $^{2-4}$

Neutral heterocyclic radicals containing sulfur/seleniumnitrogen rings, i.e., heterocyclic thiazyl/selenazyl radicals, play important roles in the development of molecule-based functional materials. ${ }^{5-13}$ In fact, there is a long-lasting interest in the charge transport ${ }^{14-17}$ and magnetic ${ }^{8,18-20}$ properties of heterocyclic thiazyl and selenazyl radicals, as the presence of heavy heteroatoms in these systems enhances both intermolecular electronic and magnetic exchange interactions. ${ }^{10,21,22}$ In such compounds, the crucial component is the conjugated $\pi$-electron system. Specifically, the interactions between the $\pi$-systems of the radicals play the key role in determining magnetic and electrical properties. Therefore, attempts to modulate the $\pi$-interactions among radicals by crystal engineering have been highly pursued. ${ }^{23-27}$ Within this context, the resonance stabilized bisdithiazolyl framework 
(a)

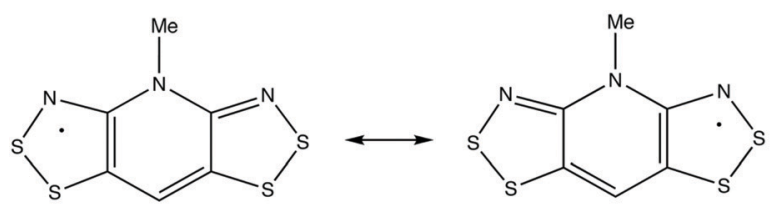

(b)

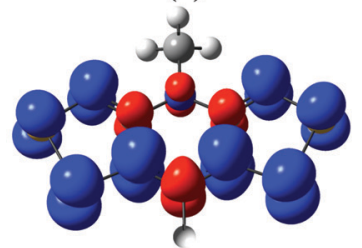

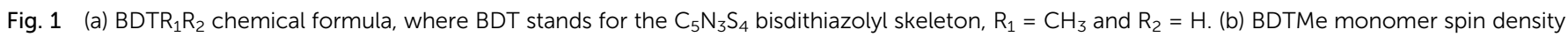
distribution (isodensity surface $=0.002$ a.u.).

BDTR $_{1} R_{2}$ (Fig. 1a) represents an appealing building block. ${ }^{28,29}$ In particular, bisdithiazolyl units have proved to be ideal candidates to address the challenge of building multi-dimensional magnetism in molecular materials, for which their exposed skeleton allows for intermolecular weak hydrogen bonds and $\pi-\pi$ interactions. $^{30}$ Modification of the axial substituents $R_{1} / R_{2}$ and the incorporation of selenium have provided a wide range of materials showing both enhanced conductivity ${ }^{12,31-34}$ and interesting magnetic properties, such as ferromagnetism, ferrimagnetism, antiferromagnetism, metamagnetic behavior, and spin-Peierls transition. ${ }^{32,35-41}$

Former experience in bistable dithiazolyl DTA ${ }^{42-46}$ and multiorbital semiquinone-bridged bisdithiazolyl $\mathrm{XBBO}^{47}$ compounds triggered us to undertake the study of the bisdithiazolyl compound BDTMe (see Fig. 1a, where $\mathrm{R}_{1}=\mathrm{CH}_{3}$ and $\mathrm{R}_{2}=\mathrm{H}$, whose systematic nomenclature is 4-methyl-4H-bis(1,2,3)dithiazolo$\left(4,5-b: 5^{\prime}, 4^{\prime}-e\right)$ pyrid-3-yl radical $\left.^{39}\right)$, since it is the first example of a thiazyl radical to exhibit metamagnetic behavior. Metamagnetism is a type of magnetic transition displayed by some low-dimensional systems (e.g. displaying chains or layers as main topological motif) with competing magnetic interactions. ${ }^{48}$ In a metamagnet, the net moments arising from intra-motif interactions are aligned antiparallel as a result of weak inter-motif antiferromagnetic (AFM) interactions to give rise to an AFM state. According to Carlin's book definition of metamagnet, the application of an external magnetic field can break up the AFM ordering and turn the system to a ferromagnetic (FM) state without going through a spin-flop state. ${ }^{48}$ The Neel temperature $\left(T_{\mathrm{N}}\right)$ for AFM ordering and the critical field $\left(H_{\mathrm{C}}\right)$ for the metamagnetic transition are sensitive to the inter-motif interactions. At this point it is fair to comment that, after metamagnetic transition, we will show that the system does not become a FM state. The key concept is instead the competition between AFM and FM interactions that will directly affect the Boltzmann population of the distinct states that belong to the magnetic topology of a given molecular material and, thus, its magnetic response.

Regarding the BDTMe radical, Oakley and co-workers have reported both magnetic measurements (down to $2 \mathrm{~K}$ ) and high quality crystal properties (at $35 \mathrm{~K}, 100 \mathrm{~K}$ and $293 \mathrm{~K}){ }^{39,49,50}$ Early magnetic susceptibility $(\chi)$ measurements indicated a significant FM surge in $\chi T$ at low temperatures. In addition, it was reported that below $5 \mathrm{~K}$ the material orders ferromagnetically or antiferromagnetically depending on the magnitude of the applied magnetic field. In this paper we have investigated the microscopic $J_{i}$ magnetic exchange interactions in BDTMe by means of a first-principles bottom-up (FPBU) ${ }^{51,52}$ procedure using $a b$ initio methods in order to understand its macroscopic magnetic behavior. Hence we use the standard static approach for both the interpretation and the simulation of the magnetic properties of the BDTMe material assuming that these properties can be obtained with a single static configuration (an X-ray resolved structure or, alternatively, an optimized structure). Therefore, using the available X-ray data, we have first characterized the magnetic topology of BDTMe in terms of all significant $J_{i}$ magnetic interactions. Surprisingly, the magnetic topology is found to be a threedimensional (3D) $J_{i}$-network and does not change drastically as a function of temperature, i.e. it is preserved irrespective of using the crystallographic data at $35 \mathrm{~K}$ or $100 \mathrm{~K}$. We have then calculated $\chi T(T)$ and $M(H)$ and compared our data to experiment in order to assess whether the BDTMe system is well described. Finally, the study of $\mathrm{d} M / \mathrm{d} H(H)$ as a function of temperature has shed light to why BDTMe behaves as a metamagnet.

\section{Computational details}

The first principles bottom-up (FPBU) working strategy follows four steps. ${ }^{51,52}$ First, one has to select all possible magnetically relevant pairs of radicals in the crystal by analysis of the crystal packing. As for the BDTMe crystal, the pairs of BDTMe radicals have been chosen in terms of the S. .S distance, since the spin density of this radical is delocalized over $\mathrm{S}, \mathrm{N}$ and $\mathrm{C}$ atoms (see Fig. 1b). Accordingly, only $i$ dimers (di) of BDTMe radicals whose $\mathrm{S}$. . S distance was shorter than a threshold distance of $8 \AA$ were selected. The choice of the BDTMe pairs has been conducted in order to include all exchange interactions that might be magnetically significant. ${ }^{53}$

It is then next required the computation of the radicalradical $J(\mathrm{di})$ interaction for each pair of radicals selected in the crystal, referred to as $J_{i}$ thorough the paper. The microscopic $J_{i}$ magnetic interaction is evaluated in terms of energy differences. The energy calculations were performed at DFT/ UB3LYP ${ }^{54,55}$ level as implemented in Gaussian. ${ }^{56}$ The standard 6-311++G(d,p) basis set ${ }^{57-61}$ was used in all energy calculations.

Once all intrinsic $J_{i}$ exchange couplings have been computed, one must propose the magnetic topology of the crystal in terms of all non-negligible $J_{i}$ magnetic interactions. In order to use Statistical Mechanics to calculate the macroscopic magnetic properties of the BDTMe crystal, one needs to select magnetic models. Specifically, the minimal magnetic model space is of 
crucial importance since it is the smallest set of $J_{i}$ interactions whose extension along $(a, b, c)$ crystallographic axes would re-generate the whole magnetic topology.

Finally, having chosen the magnetic models, we construct the matrix representation that contains all $J_{i}$ values required to appropriately parameterize the Heisenberg Hamiltonian. Those resulting parameterized matrices are then fully diagonalized on the space of spin functions of the minimal magnetic model. The energy eigenvalues and corresponding spin numbers are obtained as a result. Those values allow us to calculate the magnetic susceptibility $\chi(T)$, heat capacity $C_{\mathrm{p}}(T)$ and magnetization $M(H)$ data for each magnetic model using the corresponding expression provided by Statistical Mechanics. ${ }^{62}$ Finally the calculated data is compared to the experimentally measured data to make sure the FPBU procedure has worked correctly.

\section{Results and discussion}

The X-ray structure of the BDTMe crystal has been characterized at $35 \mathrm{~K}, 100 \mathrm{~K}$ and $293 \mathrm{~K}^{39,49,50}$ It belongs to the orthorhombic space group $\mathrm{P} 2{ }_{1} 2_{1} 2_{1}$ with unit cell parameters $a=3.882 \AA, b=$ $11.911 \AA, c=18.223 \AA$ (at $35 \mathrm{~K}$ ). The crystal packing was analyzed in terms of the shortest $\mathrm{S} \cdots \mathrm{S}$ distances between pairs of radicals. Only those $i$ dimers (di) whose minimum $\mathrm{S} \cdots \mathrm{S}$ distance was below $8 \AA$ A were selected (i.e. with centroid . . centroid distance set to $11.5 \AA$ ). Such criterion suggested 10 possible dimers as candidates to evaluate the $J_{i}$ exchange interaction between pairs of BDTMe radicals (see inset of Fig. 2 for d1-d10 pairs of radicals within the crystal, and ESI $\dagger$ Section S1 for depiction of isolated pairs of radicals and list of most relevant distances).

As mentioned in the Computational Details section, the quantitative estimation of the strength of the $J_{i}$ magnetic interactions between BDTMe radicals was carried out at unrestricted broken symmetry UB3LYP/6-311++G(d,p) level using the geometry from the experimental crystal structures at $35 \mathrm{~K}$ and $100 \mathrm{~K}$. The resulting data are in agreement with previous $a b$ initio studies performed at a different level of theory (see ESI $\uparrow$ Section S2). ${ }^{39}$ Here we must stress that experimentally the susceptibility curve dependence over the entire range of temperatures could not be fitted to any model because there is no empirical model suited for the BDTMe magnetic topology here reported. Among the ten potential interactions, only seven had relevant $J_{i}$ values (see Table 1), irrespective of the experimental $\mathrm{X}$-ray structure (see $\mathrm{ESI} \dagger$ Section $\mathrm{S} 1$ for discussion on value of $J$ magnetic interactions). The strongest calculated interactions $\left(J_{1}, J_{3}\right.$ and $\left.J_{4}\right)$ correspond to FM couplings between pairs of radicals. In particular, $J_{4}$ interactions give rise to FM $\pi$-stacks, which run along the $a$-axis (in red in Fig. 3). These FM $\pi$-stacks are then connected in zigzag by $J_{1} \mathrm{FM}$ interactions (in blue in Fig. 3), resulting in an almost isotropic spin ladder. Those spin ladders, in turn, interact ferromagnetically by means of $J_{3}$ along the $c$-axis (in green in Fig. 3), yielding a three-dimensional (3D) FM topology. Note that the weaker $J_{10}$ FM interaction (in yellow in Fig. 3) also runs along the $c$-axis. This 3D magnetic network is further coupled by three weaker AFM exchange interactions (a)

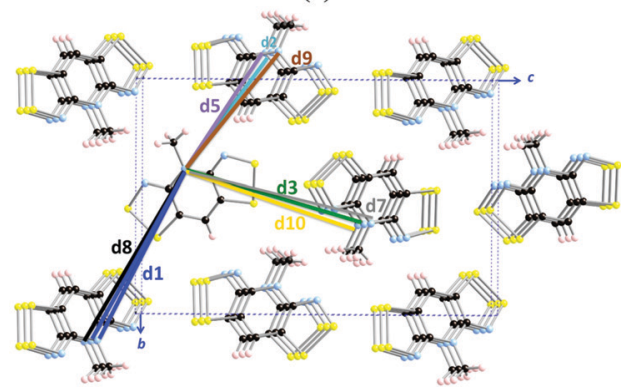

(b)

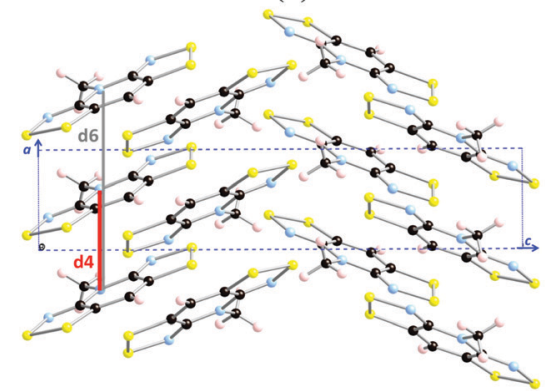

Fig. 2 Views of the crystal packing along: (a) bc plane, and (b) ac plane. Note that all d1-d10 selected pairs of BDTMe radicals are shown (MeN ... NMe contacts are explicitly depicted). Color code for $i$ dimers (di) is preserved for $J_{i}$ magnetic interactions (namely, d1 in blue, d2 in turquoise, d3 in green, d4 in red, $d 5$ in purple, $d 6-d 7$ in gray, $d 8$ in black, $d 9$ in brown, $d 10$ in yellow).

Table 1 Calculated $J_{i}$ magnetic interactions between BDTMe radicals using $35 \mathrm{~K}$ and $100 \mathrm{~K}$ crystallographic data at UB3LYP/6-311++G(d,p) level of theory

\begin{tabular}{|c|c|c|c|c|c|}
\hline Candidate di & $d($ centroid $\cdots$ centroid $) / \AA$ & $d(\mathrm{MeN} \cdots \mathrm{NMe}) / \AA$ & $d_{\min }(\mathrm{S} \cdots \mathrm{S}) / \AA$ & $J_{i}^{35 \mathrm{~K}} / \mathrm{cm}^{-1}$ & $J_{i}^{100 \mathrm{~K}} / \mathrm{cm}^{-1}$ \\
\hline $\mathrm{d} 1$ & 8.652 & 10.039 & 3.355 & 5.59 & 5.78 \\
\hline $\mathrm{d} 2$ & 6.923 & 7.521 & 3.337 & -0.61 & -1.11 \\
\hline $\mathrm{d} 3$ & 9.217 & 9.583 & 3.329 & 2.68 & 2.48 \\
\hline $\mathrm{d} 4$ & 3.882 & 3.882 & 3.779 & 8.81 & 4.83 \\
\hline d5 & 8.222 & 8.840 & 5.817 & -0.15 & -0.14 \\
\hline d6 & 7.765 & 7.765 & 7.367 & $<|0.05|$ & $<|0.05|$ \\
\hline $\mathrm{d} 7$ & 10.512 & 10.746 & 5.996 & $<0.05 \mid$ & $<|0.05|$ \\
\hline $\mathrm{d} 8$ & 10.247 & 11.442 & 6.425 & $<|0.05|$ & $<|0.05|$ \\
\hline d9 & 7.643 & 8.070 & 4.310 & -0.59 & -0.87 \\
\hline $\mathrm{d} 10$ & 9.9462 & 9.917 & 3.641 & 0.69 & -0.30 \\
\hline
\end{tabular}




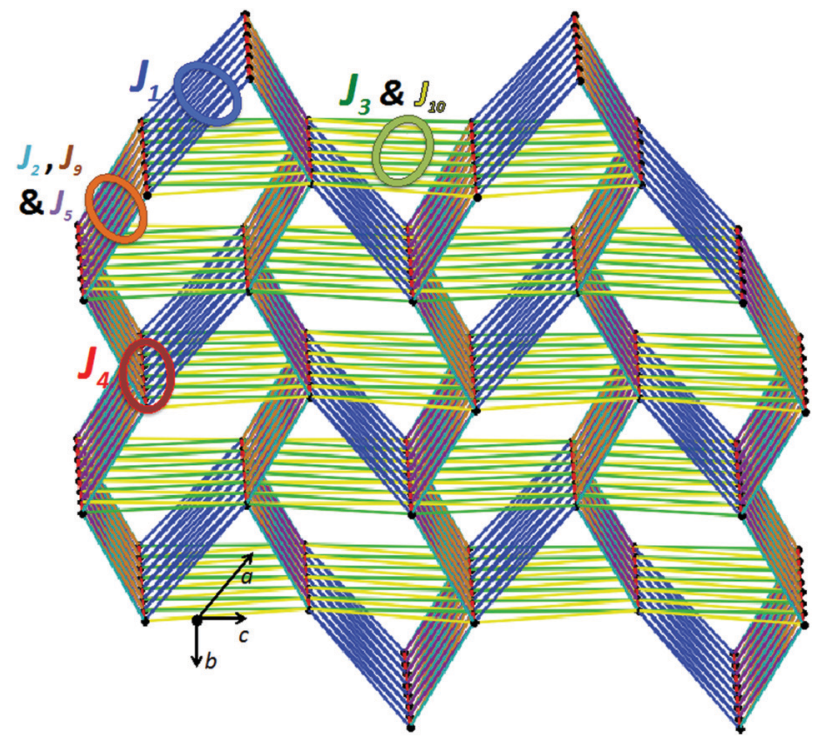

Fig. 3 View of the magnetic topology of BDTMe as a function of three dominant $F M J_{4}, J_{1}$, and $J_{3}$ interactions, and four weaker magnetic couplings: $F M J_{10}$ and AFM $J_{2}, J_{9}$ and $J_{5}$. For color code see Fig. 2 caption. Note that each BDTMe radical has been replaced by a point site.

$\left(J_{2}, J_{9}\right.$, and $J_{5}$ in turquoise, brown and purple in Fig. 3) that are one order of magnitude smaller and connect spin ladders along the $c a$. $b$-axis. Therefore, the resulting magnetic topology is a rich network of mainly FM BDTMe...BDTMe interactions. However, there are competing weaker AFM contacts that might introduce certain degree of geometrical spin frustration since they cannot possibly satisfy the corresponding AFM coupling (see Fig. 4 for a schematic representation of the spin alignment as a function of the strength of the computed $J_{i}$ exchange interactions). Let us stress here that this is not the first time we encounter this geometrical spin frustration, ${ }^{47,63}$ which was found to introduce very interesting features to the studied molecule-based systems. Further, we have proved that the magnetic topology of BDTMe is a 3D network of FM and AFM spin couplings, contrary to the common accepted idea that metamagnetism is a special type of antiferromagnetism occurring in some low-dimensional systems (e.g. displaying chains or layers as topological motif) with competing magnetic interaction. To sum up, according to our results, the $3 \mathrm{D}$ magnetic topology is qualitatively preserved at both 35 and $100 \mathrm{~K}$. Therefore, we will proceed studying the magnetic response of the BDTMe crystal using the computational data at $35 \mathrm{~K}$ since it is closer to $5 \mathrm{~K}$, below which the material orders ferromagnetically or antiferromagnetically depending on the magnitude of the applied magnetic field.

Due to the complex 3D magnetic topology of BDTMe (Fig. 3), the magnetic building block could not be easily excised. Instead, simple magnetic models were tested (see ESI $\dagger$ Section S3) in order to understand the magnetic behavior and offer a more realistic view of the complex 3D system. Eventually, two models were finally chosen (see Fig. 5a), both of them containing 16 radical molecules and the important magnetic interactions collected in Table 1 . The first $2(4+4)$ model accounts for 2 connected spin ladders to consider the effect of the number of $\pi$-stacked radicals ( $a$-axis). Each spin ladder has 4-radicals along the rail direction. The second 4(2+2) model accounts for 4 connected spin ladders and is meant to explore the cooperativity introduced by $J_{3}$. Note that previous studies showed that a 4-radical spin ladder model (i.e. with 2-radicals along the rail) was sufficient as minimal magnetic spin ladder model. $^{51,64,65}$

Comparison between the experimental data and the computed magnetic susceptibility $\chi T$ as a function of temperature for the two models using Statistical Mechanics is shown in Fig. 5b. In the experimental curve there is a rise in $\chi T$ below (a)

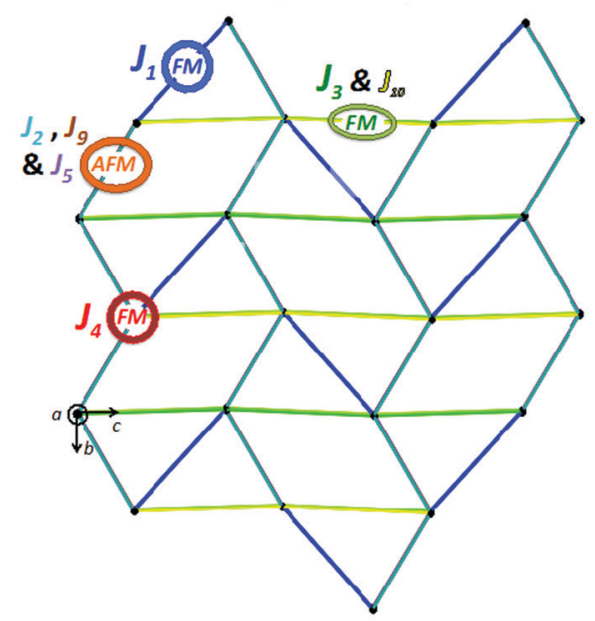

(b)

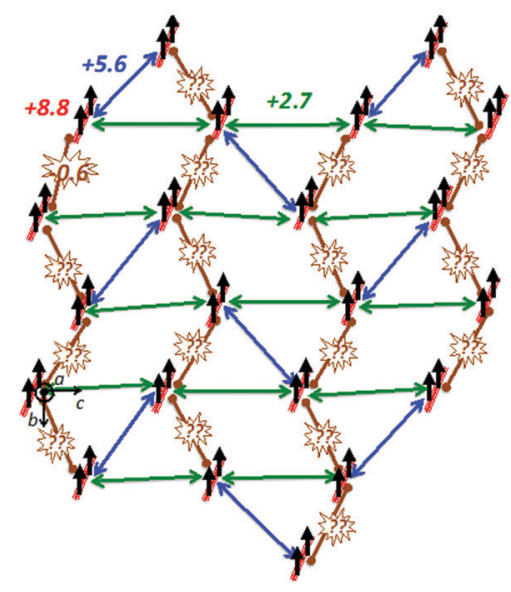

Fig. 4 (a) View of the magnetic topology parallel to the bc-plane with either FM or AFM labels highlighting the magnetic behavior of the significant exchange interactions. (b) Schematic representation of the spin alignment according to the strength of $J_{i}$ exchange interactions. The question marks illustrate the geometrical spin frustration encountered by the weakest AFM interactions. In both ( $a$ and b) the honeycomb-like connectivity of the main $J_{i}$ FM interactions between radicals is fully acknowledged. 
(a)

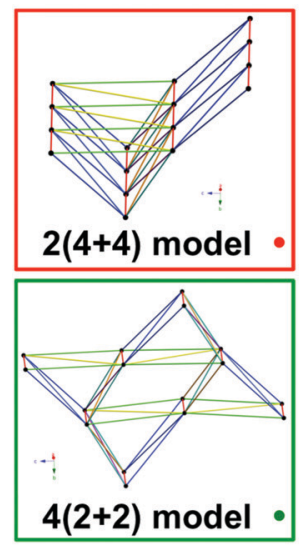

(b)

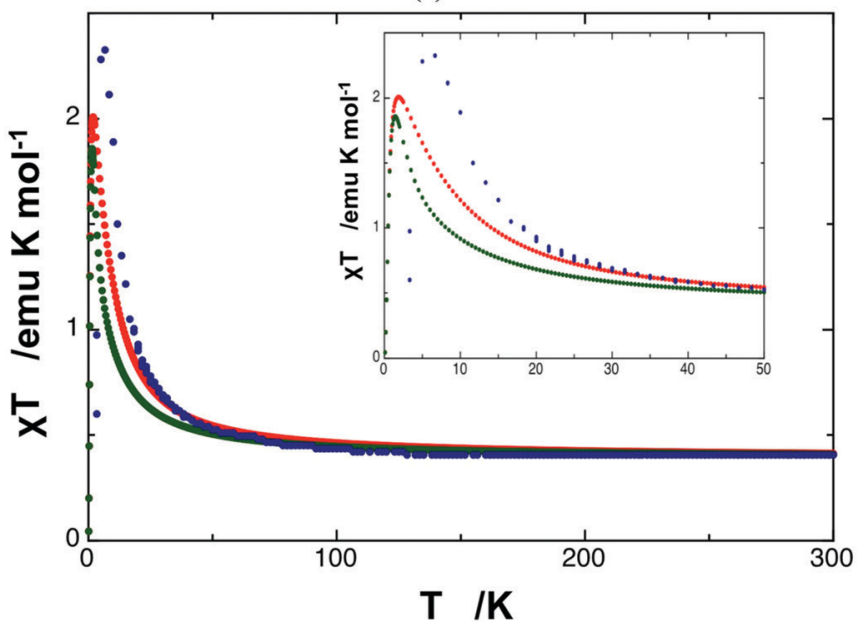

Fig. 5 (a) Magnetic $2(4+4)$ and $4(2+2)$ models. (b) $\chi T(T)$ plots at $H=0.1 T$ for the $2(4+4)$ model $($ red), the $4(2+2)$ model (green), and the experimental data (blue) for temperatures from $0-300 \mathrm{~K}$. Inset view a zoom for the $0-50 \mathrm{~K}$ range of temperatures.

$100 \mathrm{~K}$ and a sudden decrease at approximately $5 \mathrm{~K}$. This suggests a FM coupling at high temperatures and the onset of AFM interactions at low temperatures. Both models describe properly $\chi T$ at high temperatures. However, the low temperature region is not reproduced exactly. Yet, the $\chi T$ curves evidence the AFM coupling at low temperatures. Therefore, we could say that the high temperature region is described correctly, and there is a reasonable description at low temperatures. For the $2(4+4)$ model (Fig. $5 \mathrm{a}$, top), the $\pi$-stack $J_{4}$ rail (the strongest FM interaction) and $J_{1}$ rung interactions propagate. As a consequence, the $\chi T$ curve rises at higher temperatures compared to the $4(2+2)$ model (Fig. 5a, bottom). Further, according to Fig. 5b, the slope of the $\chi T$ curve using the $4(2+2)$ model resembles much more the experimental curve, although there is a temperature lag to reach the maximum $\chi T$ value. We believe that the calculated data is shifted by $c a .3 \mathrm{~K}$ due to the fact that no periodic boundary conditions could be applied to the magnetic models. Therefore, although the magnetic model has limitations, the analysis of the $\chi T$ curves enables to conclude that the $4(2+2)$ model reproduces better the experimental results.

Previous experimental work ${ }^{39}$ examined the isothermal magnetization at low temperatures for different applied magnetic fields. Below $5 \mathrm{~K}$ (the temperature at which the experimental $\chi T$ data start to drop), the magnetization was observed to increase in the region of $H=6-8$ kOe which suggested a field dependence of the magnetic ordering of the FM $\pi$-stacks switching from AFM to FM. In order to clarify these results, calculations of the magnetization dependence on the applied magnetic field for different temperatures were carried out for the $4(2+2)$ model.

In agreement with experiment, a paramagnetic behavior for $M(H)$ is observed at $T \geq 10 \mathrm{~K}$, and the $M(H)$ curve raises more rapidly for $T=6 \mathrm{~K}$ (see green and red data in Fig. 6a and b). However, for $T=2 \mathrm{~K}$ the magnetization does not reach full saturation at $1 \mathrm{~T}(H=10 \mathrm{kOe})$, as experimentally observed, but at $4 \mathrm{~T}$ (see blue data in Fig. 6a). In our simulations, full saturation at $1 \mathrm{~K}$ and $0.5 \mathrm{~K}$ is reached at $c a .2 \mathrm{~T}$ (see purple and turquoise data in Fig. $6 \mathrm{a}$ and b). It is at these temperatures that the $M(H)$ (a)

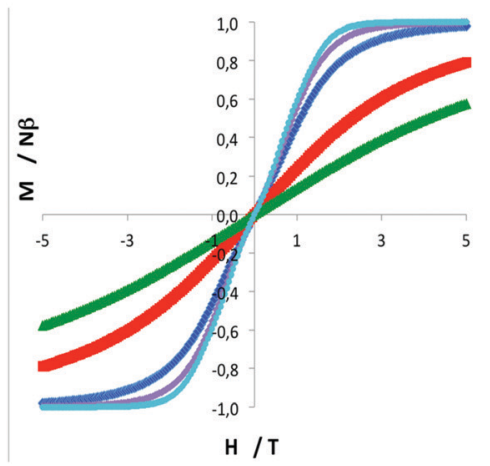

(b)

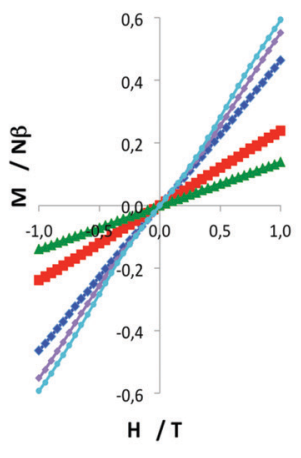

(c)

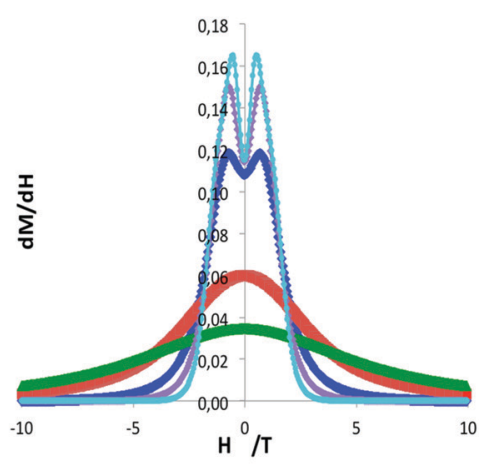

Fig. 6 For the $4(2+2)$ model, plot of the normalized magnetization as $M(H)$ (a) from $-5 T \leq H \leq 5 \mathrm{~T}$, (b) zoom in the range -1 to $+1 \mathrm{~T}$, and $(\mathrm{c}) \mathrm{d} M / \mathrm{d} H(H)$. All simulations have been performed at five different temperatures, namely at $T=0.5 \mathrm{~K}$ (light blue), $1 \mathrm{~K}$ (purple), $2 \mathrm{~K}$ (blue), $6 \mathrm{~K}$ (red), and $10 \mathrm{~K}$ (green). 
curve clearly shows the typical sigmoidal shape, where the $M(H)$ suffers a sudden increase with a small variation of the externally applied magnetic field. This feature became more apparent in the plot of $\mathrm{d} M / \mathrm{d} H$ versus $H$ (see Fig. 6c), in which the critical field is found to be $c a$. $0.7 \mathrm{~T}\left(H_{\mathrm{C}}=7 \mathrm{kOe}\right.$ instead of the experimental $6 \mathrm{kOe})$. Note that $H_{\mathrm{C}}$ is estimated as an average of the field at which a maximum $\mathrm{d} M / \mathrm{d} H$ value is reached. We must stress that this behavior is a classic signature of a metamagnetic system for the region between $H=0-1 \mathrm{~T}^{62,66-72} \mathrm{We}$ are convinced that the estimated $H_{\mathrm{C}}$ data does not quantitatively reproduce the experimental value due to the fact that no periodic boundary conditions could be applied to the magnetic models.

Experimentally, below $6 \mathrm{~K}$ and with low applied magnetic fields, it was suggested that the metamagnetic-like transition in BDTMe results from the presence of small AFM interactions among the radical $\pi$-stacks which could be considered as $1 \mathrm{D}$ FM $S=1 / 2$ chains. At higher fields (above $6 \mathrm{kOe}$ ), the direction of the magnetization of alternate chains was found to be reversed by the field. Specifically, all the FM chains became aligned in parallel, thereby producing a ferromagnetically ordered system. Yet we know that the magnetic topology of BDTMe does not consist of FM chains but of a 3D FM network. Therefore, the metamagnetic behavior of BDTMe is related to the presence of AFM interaction within the 3D FM topology of radicals. Also we have worked the magnetic topology out at $35 \mathrm{~K}$ and $100 \mathrm{~K}$, and concluded that it is qualitatively preserved irrespective of the BDTMe characterization temperature. Accordingly, since the magnetic topology is the same for temperatures below $35 \mathrm{~K}$, the ground state GS must also be the same irrespective of the temperature at which we study the BDTMe crystal. Therefore, revising the commonly accepted picture of a metamagnet explained as GS changing from AFM to FM, we here propose that it is the Boltzmann population of the different states the key concept: at $2 \mathrm{~K}$ the ground singlet $S=0$ state has more weight $(24 \%)$ than at $10 \mathrm{~K}(1.5 \%)$. Therefore at $10 \mathrm{~K}$ there is a very large number of excited spin states populated of multiplicity either different than $S=0$ (e.g. triplet, quartet, quintuplet, etc.) or alike. Let us stress here that the magnetic behavior of the charge-transfer (HMTTF) [Ni(mnt $\left.)_{2}\right]$ material was also rationalized solely in terms of the different Boltzmann population of diamagnetic and paramagnetic spin states as the temperature changes. ${ }^{73}$ For BDTMe, the difference in populations translates into the metamagnetic transition. All these data enable us to conclude that changes in the AFM values of $J_{2}, J_{5}$ and $J_{9}$ will directly affect the population of the distinct states that belong to a given magnetic topology. It thus follows that the magnetic response of the BDTMe moleculebased metamagnet can be tuned by means of weak AFM interactions, which we have fully identified. One straightforward way to proceed could be to make minor modifications on the peripheral groups of the bisdithiazolyl unit, investigate the new magnetic responses at low temperature, and analyze how critically does the magnetism and the crystal packing depend on the chemical modifications. However appealing, the manipulation of these AFM interactions is at the very least a massive challenge that goes beyond the scope of this paper.

\section{Conclusions}

The BDTMe crystal is unveiled to display a 3D magnetic topology, contrary to the common idea that metamagnetism occurs in lowdimensional system. For BDTMe, the complex 3D network is mainly FM and consists of almost isotropic FM spin-ladders (whose rails are $\pi$-stacks of BDTMe radicals along the $a$-axis) coupled ferromagnetically (along the $c$-axis), irrespective of the characterization temperature. Besides, some weaker AFM interactions are found within the crystal packing, which are demonstrated to be crucial to understand the magnetic response of the BDTMe molecule-based crystal.

Different magnetic models have been tested in order to reproduce the macroscopic magnetic properties of the crystal (namely, magnetic susceptibility $\chi$ and magnetization $M$ as a function of temperature and field, respectively). As a result, the $\chi T(T)$ curve was not only fully reproduced at high temperatures, but also reasonably well described at low temperatures. In the latter region, our simulations predict a $c a .3 \mathrm{~K}$ shift towards lower temperatures compared to experiment referring to the temperature at which the calculated $\chi T(T)$ data is maximum. Comparison of calculated and experimental $M(H)$ data enables to conclude that the $M(H)$ curve clearly shows the typical sigmoidal shape of a metamagnet at temperatures below ca. $2 \mathrm{~K}$. This feature becomes more apparent in the plot of $\mathrm{d} M / \mathrm{d} H(H)$, in which the critical field is found to be $c a .0 .7 \mathrm{~T}$ (7 kOe) in very good agreement with the experimental $6 \mathrm{kOe}$.

The magnetic topology does not depend on the BDTMe characterization temperature. Accordingly, the ground state is the same irrespective of the temperature at which we study the BDTMe crystal. Revising the commonly accepted understanding of a metamagnet explained as ground state changing from AFM to FM, the Boltzmann population of the different states is suggested to be the key concept: at $2 \mathrm{~K}$ the ground singlet state has more weight (24\%) than at $10 \mathrm{~K}(1.5 \%)$. This difference in populations translates into the metamagnetic transition for BDTMe molecule-based material. Our data enable us to conclude that changes in the AFM interactions that couple the 3D FM topology will directly affect the population of the distinct states that belong to a given magnetic topology and, in turn, its magnetic response.

From our study, one infers that the metamagnetic behavior in bisdithiazolyl-based materials may be due to the interchain weak interactions, which in the BDTMe case were inter-spinladder weak AFM couplings. We believe that this strategy could be valid for a wide range of similar sulfur-nitrogen heterocyclic ring compounds whose magnetism can be thus tuned by means of weak AFM interactions. It is therefore exceedingly important to determine the role of weak interactions over the magnetic behavior of a molecule-base material.

\section{Conflicts of interest}

There are no conflicts of interest to declare.

\section{Acknowledgements}

Financial support from MINECO MAT2014-54025-P and CTQ201787773-P/AEI/FEDER projects, Spanish Structures of Excellence 
María de Maeztu program through grant MDM-2017-0767, and Catalan DURSI for projects 2014SGR1422 and 2017SGR348 are fully acknowledged. JJS acknowledges funding from the European Union Horizon 2020 research and innovation program under the Marie Sklodowska-Curie Grant Agreement No. 795246-StrongLights.

\section{References}

1 J. S. Miller and D. Gatteschi, Molecule-based Magnets (themed issue), Chem. Soc. Rev., 2011, 40, 3053-3368.

2 G. Aromí, D. Aguilà, P. Gamez, F. Luis and O. Roubeau, Chem. Soc. Rev., 2012, 41, 537-546.

3 J. Ferrando-Soria, J. Vallejo, M. Castellano, J. Martinez-Lillo, E. Pardo, J. Cano, L. Castro, F. Lloret, R. Ruiz-Garcia and M. Julve, Coord. Chem. Rev., 2017, 339, 17-103.

4 G. Mínguez Espallargas and E. Coronado, Chem. Soc. Rev., 2018, 47, 533-557.

5 T. Chivers, Guide to Chalcogen-Nitrogen Chemistry, World Scientific, 2005.

6 F. Palacio, G. Antorrena, M. Castro, R. Burriel, J. M. Rawson, J. N. B. Smith, N. Bricklebank, J. J. Novoa and C. Ritter, Phys. Rev. Lett., 1997, 79, 2336-2339.

7 T. M. Barclay, A. W. Cordes, N. A. George, R. C. Haddon, M. E. Itkis, M. S. Mashuta, R. T. Oakley, G. W. Patenaude, R. W. Reed, J. F. Richardson and H. Zhang, J. Am. Chem. Soc., 1998, 120, 352-360.

8 W. Fujita and K. Awaga, Science, 1999, 286, 261-262.

9 K. E. Preuss, Dalton Trans., 2007, 2357-2369.

10 J. M. Rawson, A. Alberola and A. Whalley, J. Mater. Chem., 2006, 16, 2560-2575.

11 L. Beer, J. L. Brusso, R. C. Haddon, M. E. Itkis, R. T. Oakley, R. W. Reed, J. F. Richardson, R. A. Secco and X. Y. Yu, Chem. Commun., 2005, 5745-5747.

12 J. L. Brusso, K. Cvrkalj, A. A. Leitch, R. T. Oakley, R. W. Reed and C. M. Robertson, J. Am. Chem. Soc., 2006, 128, 15080-15081.

13 O. A. Rakitin and A. V. Zibarev, Asian J. Org. Chem., 2018, 7, 2397-2416.

14 A. W. Cordes, R. C. Haddon and R. T. Oakley, Adv. Mater., 1994, 6, 798-802.

15 T. M. Barclay, A. W. Cordes, R. C. Haddon, M. E. Itkis, R. T. Oakley, R. W. Reed and H. Zhang, J. Am. Chem. Soc., 1999, 121, 969-976.

16 D. Tian, S. M. Winter, A. Mailman, J. W. L. Wong, W. Yong, H. Yamaguchi, Y. Jia, J. S. Tse, S. Desgreniers, R. A. Secco, S. R. Julian, C. Jin, M. Mito, Y. Ohishi and R. T. Oakley, J. Am. Chem. Soc., 2015, 137, 14136-14148.

17 H. Oberhofer, K. Reuter and J. Blumberger, Chem. Rev., 2017, 117, 10319-10357.

18 A. J. Banister, N. Bricklebank, I. Lavender, J. M. Rawson, C. I. Gregory, B. K. Tanner, W. Clegg, M. R. Elsegood and F. Palacio, Angew. Chem., Int. Ed. Engl., 1996, 35, 2533-2535.

19 J. L. Brusso, O. P. Clements, R. C. Haddon, M. E. Itkis, A. A. Leitch, R. T. Oakley, R. W. Reed and J. F. Richardson, J. Am. Chem. Soc., 2004, 126, 8256-8265.
20 D. Bates, C. M. Robertson, A. A. Leitch, P. A. Dube and R. T. Oakley, J. Am. Chem. Soc., 2018, 140, 3846-3849.

21 R. G. Hicks, Org. Biomol. Chem., 2007, 5, 1321-1338.

22 F. Breher, Coord. Chem. Rev., 2007, 251, 1007-1043.

23 J. M. Lu, S. V. Rosokha and J. K. Kochi, J. Am. Chem. Soc., 2003, 125, 12161-12171.

24 T. Devic, M. Yuan, J. Adams, D. C. Fredrickson, S. Lee and D. Venkataraman, J. Am. Chem. Soc., 2005, 127, 14616-14627.

25 V. Podzorov, Nat. Mater., 2010, 9, 616-617.

26 R. G. Hicks, Nat. Chem., 2011, 3, 189-191.

27 K. Molcanov, B. Kojic-Prodic, D. Babic, D. Pajic, N. Novosel and K. Zadro, CrystEngComm, 2012, 14, 7958-7964.

28 L. Beer, J. L. Brusso, A. W. Cordes, R. C. Haddon, M. E. Itkis, K. Kirschbaum, D. S. MacGregor, R. T. Oakley, A. A. Pinkerton and R. W. Reed, J. Am. Chem. Soc., 2002, 124, 9498-9509.

29 A. W. Cordes, R. C. Haddon and R. T. Oakley, Phosphorus, Sulfur Silicon Relat. Elem., 2004, 179, 673-684.

30 Y. Shuku, Y. Hirai, N. A. Semenov, E. Kadilenko, N. P. Gritsan, A. V. Zibarev, O. A. Rakitin and K. Awaga, Dalton Trans., 2018, 27, 9897-9902.

31 A. A. Leitch, X. Yu, S. M. Winter, R. A. Secco, P. A. Dube and R. T. Oakley, J. Am. Chem. Soc., 2009, 131, 7112-7125.

32 X. Yu, A. Mailman, P. A. Dube, A. Assoud and R. T. Oakley, Chem. Commun., 2011, 47, 4655-4657.

33 J. W. L. Wong, A. Mailman, S. M. Winter, C. M. Robertson, R. J. Holmberg, M. Murugesu, P. A. Dube and R. T. Oakley, Chem. Commun., 2014, 50, 785-787.

34 A. Mailman, A. A. Leitch, W. Yong, E. Steven, S. M. Winter, R. C. M. Claridge, A. Assoud, J. S. Tse, S. Desgreniers, R. A. Secco and R. T. Oakley, J. Am. Chem. Soc., 2017, 139, 2180-2183.

35 A. A. Leitch, J. L. Brusso, K. Cvrkalj, R. W. Reed, C. M. Robertson, P. A. Dube and R. T. Oakley, Chem. Commun., 2007, 3368-3370.

36 C. M. Robertson, D. J. T. Myles, A. A. Leitch, R. W. Reed, B. M. Dooley, N. L. Frank, P. A. Dube, L. K. Thompson and R. T. Oakley, J. Am. Chem. Soc., 2007, 129, 12688-12689.

37 C. M. Robertson, A. A. Leitch, K. Cvrkalj, R. W. Reed, D. J. T. Myles, P. A. Dube and R. T. Oakley, J. Am. Chem. Soc., 2008, 130, 8414-8425.

38 C. M. Robertson, A. A. Leitch, K. Cvrkalj, D. J. T. Myles, R. W. Reed, P. A. Dube and R. T. Oakley, J. Am. Chem. Soc., 2008, 130, 14791-14801.

39 S. M. Winter, K. Cvrkalj, P. A. Dube, C. M. Robertson, M. R. Probert, J. A. K. Howard and R. T. Oakley, Chem. Commun., 2009, 7306-7308.

40 K. Lekin, S. M. Winter, L. E. Downie, X. Bao, J. S. Tse, S. Desgreniers, R. A. Secco, P. A. Dube and R. T. Oakley, J. Am. Chem. Soc., 2010, 132, 16212-16224.

41 N. J. Yutronkie, D. Bates, P. A. Dube, S. M. Winter, C. M. Robertson, J. L. Brusso and R. T. Oakley, Inorg. Chem., 2019, 58, 419-427.

42 C. S. Clarke, J. Jornet, M. Deumal and J. J. Novoa, Polyhedron, 2009, 28, 1614-1619.

43 C. S. Clarke, J. Jornet-Somoza, F. Mota, J. J. Novoa and M. Deumal, J. Am. Chem. Soc., 2010, 132, 17817-17830. 
44 S. Vela, A. Sopena, J. Ribas-Arino, J. J. Novoa and M. Deumal, Chem. - Eur. J., 2014, 20, 7083-7090.

45 S. Vela, M. Deumal, M. Shiga, J. J. Novoa and J. Ribas-Arino, Chem. Sci., 2015, 6, 2371-2381.

46 T. Francese, J. Ribas-Arino, J. J. Novoa, R. W. A. Havenith, R. Broer, C. de Graaf and M. Deumal, Phys. Chem. Chem. Phys., 2018, 20, 20406-20416.

47 M. Fumanal and M. Deumal, Phys. Chem. Chem. Phys., 2016, 18, 20738-20749.

48 R. L. Carlin, Magnetochemistry, Springer, Berlin-Heidelberg, 1986. See p. 202 for definition of metamagnetism and p. 134 for definition of spin-flop.

49 L. Beer, J. L. Brusso, A. W. Cordes, E. Goodde, R. C. Haddon, M. E. Itkis, R. T. Oakley and R. W. Reed, Chem. Commun., 2002, 2562-2563.

50 L. Beer, J. F. Britten, J. L. Brusso, A. W. Cordes, R. C. Haddon, M. E. Itkis, D. S. MacGregor, R. T. Oakley, R. W. Reed and C. M. Robertson, J. Am. Chem. Soc., 2003, 125, 14394-14403.

51 M. Deumal, M. J. Bearpark, J. J. Novoa and M. A. Robb, J. Phys. Chem. A, 2002, 106, 1299-1315.

52 J. J. Novoa, M. Deumal and J. Jornet-Somoza, Chem. Soc. Rev., 2011, 40, 3182-3212.

53 Former studies ref. 39 missed some of the interactions herein taken into account.

54 A. D. Becke, Phys. Rev. A: At., Mol., Opt. Phys., 1988, 38, 3098-3100.

55 C. Lee, W. Yang and R. G. Parr, Phys. Rev. B: Condens. Matter Mater. Phys., 1988, 37, 785-789.

56 M. J. Frisch, G. W. Trucks, H. B. Schlegel, G. E. Scuseria, M. A. Robb, J. R. Cheeseman, J. A. Montgomery, Jr., T. Vreven, K. N. Kudin, J. C. Burant, J. M. Millam, S. S. Iyengar, J. Tomasi, V. Barone, B. Mennucci, M. Cossi, G. Scalmani, N. Rega, G. A. Petersson, H. Nakatsuji, M. Hada, M. Ehara, K. Toyota, R. Fukuda, J. Hasegawa, M. Ishida, T. Nakajima, Y. Honda, O. Kitao, H. Nakai, M. Klene, X. Li, J. E. Knox, H. P. Hratchian, J. B. Cross, V. Bakken, C. Adamo, J. Jaramillo, R. Gomperts, R. E. Stratmann, O. Yazyev, A. J. Austin, R. Cammi, C. Pomelli, J. W. Ochterski, P. Y. Ayala, K. Morokuma, G. A. Voth, P. Salvador, J. J. Dannenberg, V. G. Zakrzewski, S. Dapprich, A. D. Daniels, M. C. Strain, O. Farkas, D. K. Malick, A. D. Rabuck, K. Raghavachari, J. B. Foresman, J. V. Ortiz, Q. Cui, A. G. Baboul, S. Clifford, J. Cioslowski, B. B. Stefanov, G. Liu, A. Liashenko, P. Piskorz, I. Komaromi, R. L. Martin, D. J. Fox,
T. Keith, M. A. Al-Laham, C. Y. Peng, A. Nanayakkara, M. Challacombe, P. M. W. Gill, B. Johnson, W. Chen, M. W. Wong, C. Gonzalez and J. A. Pople, Gaussian 03, Revision C.02, Gaussian, Inc., Wallingford CT, 2004.

57 W. J. Hehre, R. Ditchfield and J. A. Pople, J. Chem. Phys., 1972, 56, 2257-2261.

58 P. C. Hariharan and J. A. Pople, Theor. Chim. Acta, 1973, 28, 213-222.

59 A. D. McLean and G. S. Chandler, J. Chem. Phys., 1980, 72, 5639-5648.

60 M. M. Francl, W. J. Pietro, W. J. Hehre, J. S. Binkley, D. J. DeFrees, J. A. Pople and M. S. Gordon, J. Chem. Phys., 1982, 77, 3654-3665.

61 M. J. Frisch, J. A. Pople and J. S. Binkley, J. Chem. Phys., 1984, 80, 3265-3269.

62 O. Kahn, Molecular Magnetism, VCH Publishers, New York, 1993, p. 212 for same derivation applied to dinuclear compounds.

63 S. N. Herringer, M. Deumal, J. Ribas-Arino, J. J. Novoa, C. P. Landee, J. L. Wikaira and M. M. Turnbull, Chem. Eur. J., 2014, 20, 8355-8363.

64 M. Deumal, G. Giorgi, M. A. Robb, M. M. Turnbull, C. P. Landee and J. J. Novoa, Eur. J. Inorg. Chem., 2005, 4697-4706.

65 A. Shapiro, C. P. Landee, M. M. Turnbull, J. Jornet, M. Deumal, J. J. Novoa, M. A. Robb and W. Lewis, J. Am. Chem. Soc., 2007, 129, 952-959.

66 K. Togashi, R. Imachi, K. Tomioka, H. Tsuboi, T. Ishida, T. Nogami, N. Takeda and M. Ishikawa, Bull. Chem. Soc. Jpn., 1996, 69, 2821-2830.

67 J. P. Sutter, A. Lang, O. Kahn, C. Paulsen, L. Ouahab and Y. Pei, J. Magn. Magn. Mater., 1997, 171, 147-152.

68 Magnetism: Molecules to Materials, ed. J. S. Miller and M. Drillon, Wiley-VCH, Weinheim, 2001, vol. 2.

69 S. Rabaca, R. Meira, L. C. J. Pereira, M. T. Duarte, J. J. Novoa and V. Gama, Inorg. Chim. Acta, 2001, 326, 89-100.

70 H. Nagashima, S. Fujita, H. Inoue and N. Yoshioka, Cryst. Growth Des., 2004, 4, 19-21.

71 A. Das, G. M. Rosair, M. S. El Fallah, J. Ribas and S. Mitra, Inorg. Chem., 2006, 45, 3301-3306.

72 D. Belo, J. Mendonça, I. C. Santos, L. C. J. Pereira, M. Almeida, J. J. Novoa, C. Rovira, J. Veciana and V. Gama, Eur. J. Inorg. Chem., 2008, 5327-5337.

73 S. Vela, M. Fumanal and M. Deumal, Dalton Trans., 2015, 44, 608-614. 